Денисов Александр Михайлович

старший преподаватель кафедры организационно-кадровой работы в органах государственной власти МИРЭА -

Российского технологического университета

\section{АНАЛИЗ ВЗАИМОСВЯЗИ ТЕОРИЙ МОТИВАЦИИ И ГЕЙМИФИКАЦИИ В ПРОЦЕССЕ УПРАВЛЕНИЯ ПЕРСОНАЛОМ ОРГАНИЗАЦИИ}

\begin{abstract}
Аннотация:
Статья посвящена научному, кросс-функциональному взаимодействию двух областей знания: мотивации и геймификации. В то время как каждая из областей включает в себя достаточно обиирную базу знаний, связь между ними изучена слабо. Несмотря на то, что сущность и влияние различных элементов мотивации на поведение индивидуума в социуме и, в частности, в рабочей среде знакомы исследователям достаточно давно, в арсенале мотивационных стимулов у современного руководителя остается все меньше инструментов для регулирования поведения работников в интересах организации. Исследования показывают, что в части компаний падение прибыли может быть связано не только и не столько с отсутствием заинтересованности у клиентов как потребителей товаров или услуг. Истинной причиной проблем становится недостаточная ориентированность персонала на эффективность работы. Как оказалось, жесткие системы мотивации, дисциплина и строгая отчетность не всегда дают ожидаемый результат. Рост будет, если пробудить в сотрудниках истинный интерес к рабочему процессу. Все чаще в последнее время руководители организаций и их подразделений вводят элементы геймификации в процесс управления персоналом, чтобы мотивировать работников на выполнение профессиональных задач и повысить их производительность труда. Многие менеджеры высшего звена уже признали, что геймификация стала одной из самых эффективных методик мотивации персонала. Авторы постарались раскрыть содержание термина «геймификация», рассмотреть вопросы значимости и особенностей применения данного метода мотивации и стимулирования персонала; изучить его влияние на повышение производительности труда работников и эффективности деятельности всей организации. Был проведен анализ существующих моделей мотивационных теорий с точки зрения использования в них основных компонентов геймификации; подтверждена необходимость тиательного подбора той или иной теории мотивации для реализации повышения эффективности персонала в современной организации.
\end{abstract}

Ключевые слова:

мотивация, мотив, теории мотивации, геймификация, коллектив, персонал, менеджер, управление трудовым коллективом, управление персоналом организации.
Denisov Aleksandr Mikhailovich

Senior Lecturer of the Department of Organizational and Personnel Work for Public Authorities, Russian Technological University

\section{ANALYSIS OF THE RELATIONSHIP BETWEEN MOTIVATION AND GAMIFICATION THEORIES IN THE PROCESS OF PERSONNEL MANAGEMENT IN ORGANIZATIONS}

Summary:

This study reviews the scientific, cross-functional interaction of two areas of knowledge: motivation and gamification. While each area includes a fairly extensive knowledge base, the relationship between them is poorly understood. Although the essence and influence of various elements of motivation on the behavior of an individual in society and in the working environment, in particular, has been familiar to researchers for a long time, a modern manager has fewer motivational tools for regulating the behavior of employees that could benefit the organization. The studies show that in some companies, the drop in profits may be due not only and not so much to the lack of interest among customers as consumers of goods or services. The real reason for the problems is the lack of stuff's interest in work efficiency. As it turned out, strict motivation systems, discipline and strict reporting do not always give the expected result. Improving will occur if employees are truly interested in the process. Recently, more and more managers of organizations and their departments are introducing gamification elements into the personnel management process in order to motivate employees to perform tasks and increase their productivity. Many senior managers have already recognized that gamification has become one of the most effective methods of motivating staff. The study has presented the content of the term gamification, and also considered the importance and application of gamification for motivating and stimulating staff; also studied the effect on increasing productivity of workers and the efficiency of the entire organization. Models of motivational theories are analyzed in the context of using the main components of gamification and the necessity for a thorough analysis of the approach before applying the most appropriate theory of motivation for building in-house game mechanics in modern organizations.

Keywords: motivation, motive, motivation theories, gamification, team, personnel, manager, workforce management, organization personnel management. 
Геймификация - это использование игровых элементов в неигровых системах и ситуациях, в том числе и в работе организации - для улучшения взаимодействия между сотрудниками, которое должно обеспечить достаточно высокий уровень мотивации, лояльности и повышение уровня удовлетворенности трудом [1, с. 2426].

В последние годы геймификация стала применяться в маркетинге, а также в таких сфрерах общества, как политика, здравоохранение, информационные технологии и образование [2, с. 323]. Быстрое внедрение элементов геймификации в эти сферы привлекло к себе интерес исследователей с точки зрения изучения практической пользы данного явления для системы управления персоналом в организации, что в дальнейшем может и должно привести к созданию благоприятного имиджа работодателя, привлекательного образа рабочего места и повышению интереса у сотрудника к выполняемым задачам в процессе труда.

Однако проблема состоит в том, что геймификация является предметом споров и критики со стороны некоторых влиятельных исследователей, подчеркивающих необходимость разработки таких ее систем, которые позволяли бы активизировать внутреннюю мотивацию сотрудника для более эфффективной работы, а не заменяли бы ее внешними элементами поощрения, такими как накопление иллюзорных баллов, получение реальных или виртуальных отличительных знаков и достижений [3, с. 19].

Геймификация пытается использовать мотивационную составляющую игровых ситуаций для активизации настойчивости и стремления к достижениям у людей, участвующих в этом процессе. Однако мы считаем, что следует пересмотреть понимание того, как именно повышать и поддерживать мотивацию персонала, в том числе с использованием таких инструментов геймификации, как достижения и вознаграждения.

Идея использования игровой механики для стимулирования вовлеченности работников в процесс труда за счет использования внешней мотивации, на наш взгляд, заслуживает рассмотрения. Кроме того, следует учитывать результаты ряда научных исследований, обнаруживших, что использование внешних наград может оказать значительное негативное влияние на мотивацию сотрудников, подрывая свободу выбора и снижая интерес к рабочей задаче [4, с. 31].

Для углубления понимания механизма возникновения мотивации у работников к участию в игре мы предлагаем рассмотреть теоретическую модель, содержащую целый спектр теорий мотивации и их интерпретацию в свете использования приемов геймификации.

Мотивация к действию является предметом изучения в исследованиях по социальной психологии, педагогике, менеджменту. Эти области сосредоточены на анализе специфики мотивации в конкретных сферах деятельности. Для успешного создания и использования систем геймификации мы считаем необходимым связать ее с теориями мотивации и таким образом изучить мотивационную модель поведения работников в играх.

Мотивация проявляется в выборе индивидом того или иного типа поведения, интенсификации усилий и демонстрации целеустремлённости в ходе деятельности за счет удовлетворения собственных социальных потребностей [5, с. 381].

Современные подходы к мотивации касаются двух доминирующих кластеров, определяющих заинтересованность в деятельности любого человека, в том числе участника игры. Речь идет о внутренней и внешней стороне влияния мотивации. Геймификация сочетает в себе эти два компонента.

Так, использование в процессе игры внешних поощрительных элементов (уровней, очков и т. д.) с целью стимуляции вовлеченности и формирования ощущения сопричастности к общей деятельности является средством побуждения к достижению определенного уровня мастерства, осознанию собственной независимости и возникновению чувства принадлежности к различным игровым группам, следствием чего становится выраженная социальная динамика [6, с. 324].

Социальный аспект геймификации также очень важен в играх. Конкуренция, социальное взаимодействие или сотрудничество могут влиять на поведение игроков. Опираясь на исследование Ж.Б. Васильевой, можно утверждать, что данный подход охватывает целый спектр теорий мотиваций, которые могут быть использованы для создания мотивационной модели поведения в играх [7, с. 180].

На одной стороне модели расположилась внешняя мотивация, которая находится в центре внимания теории ожидаемой полезности и теории подкрепления Скиннера. Эти теории объясняют мотивацию к совершению действий или поведению, которые обусловлены внешним вознаграждением.

На другой стороне модели находится теория потребностей Маслоу, теория достижения потребностей Аткинсона, а также теории целеполагания. Все это - теории, основанные на потребностях. 
Теории же, занявшие место в середине модели, объясняют социальную составляющую мотивации игр. В этом контексте мы рассматриваем теорию сравнения и теорию личных инвестиций (PIT) (рисунок 1).

\section{Теория самоопределения личности}

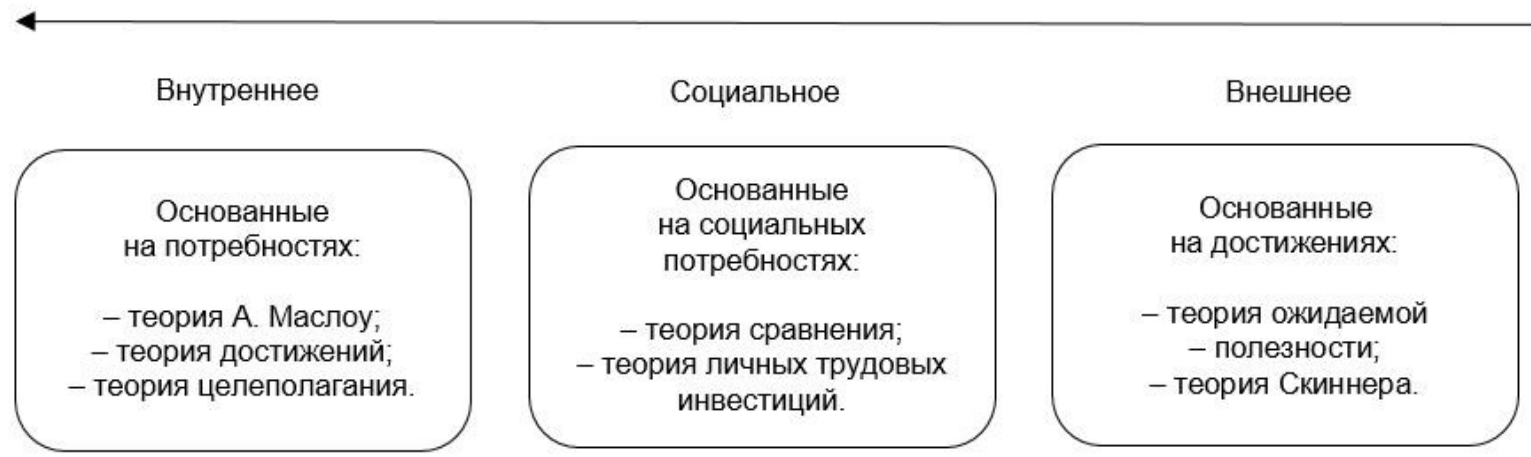

Рисунок 1 - Модель мотивации в играх

Одна из самых ранних и наиболее известных теорий мотивации исходит из психологии Абрахама Маслоу [5, с. 371]. Согласно ей, человеческим поведением движет желание удовлетворить собственные фризические и психологические потребности. Маслоу предлагает пирамидальную модель пяти уровней потребностей, которые управляют человеческой деятельностью, начиная от фризиологических потребностей и заканчивая потребностью в самоактуализации (рисунок 2).

По мере продвижения вверх по иерархии Маслоу, мы переходим от потребностей, вызываемых недостатками, к потребностям, порождаемым позитивными целями и стимулами.

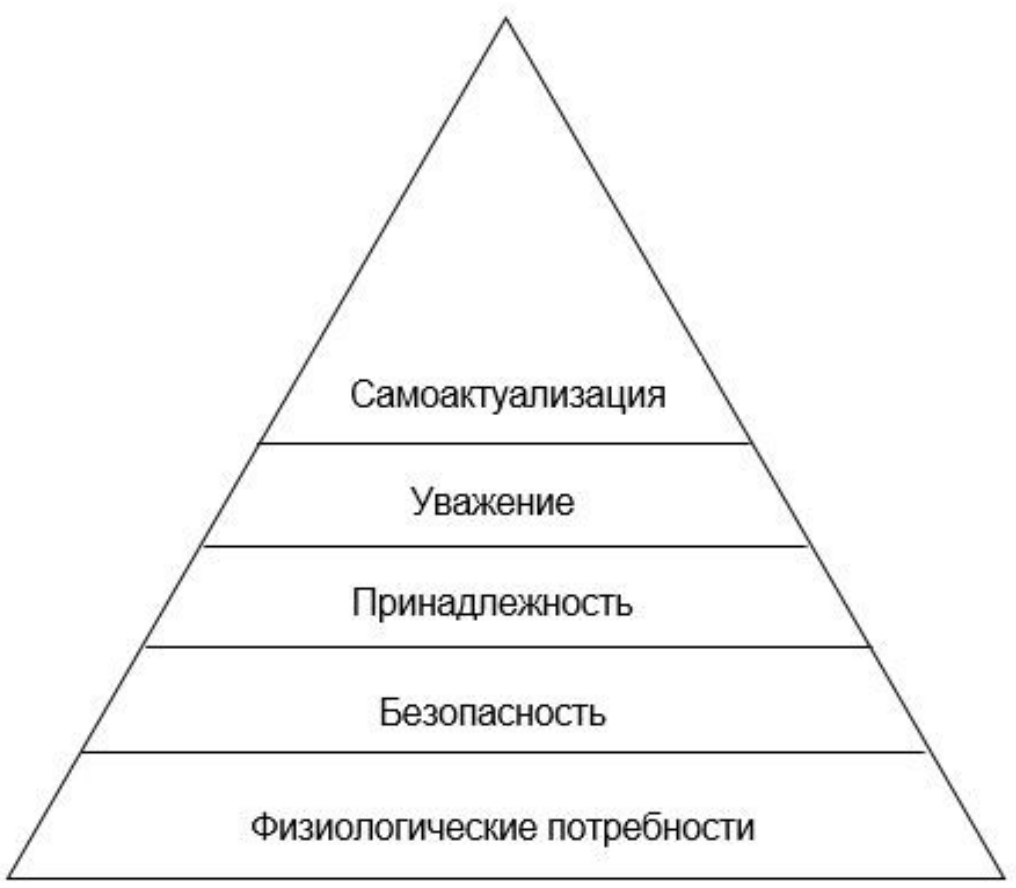

\section{Рисунок 2 - Пирамидальная модель теории мотивации Абрахама Маслоу}

Основываясь на иерархии потребностей Маслоу, Сианг иллюстрирует мотивационные потребности игроков в процессе игры, где более низкие уровни должны быть достигнуты раньше, чем возникнет возможность перехода на более высокие уровни в пирамидальной модели (рисунок 3) [8, с. 241]. 


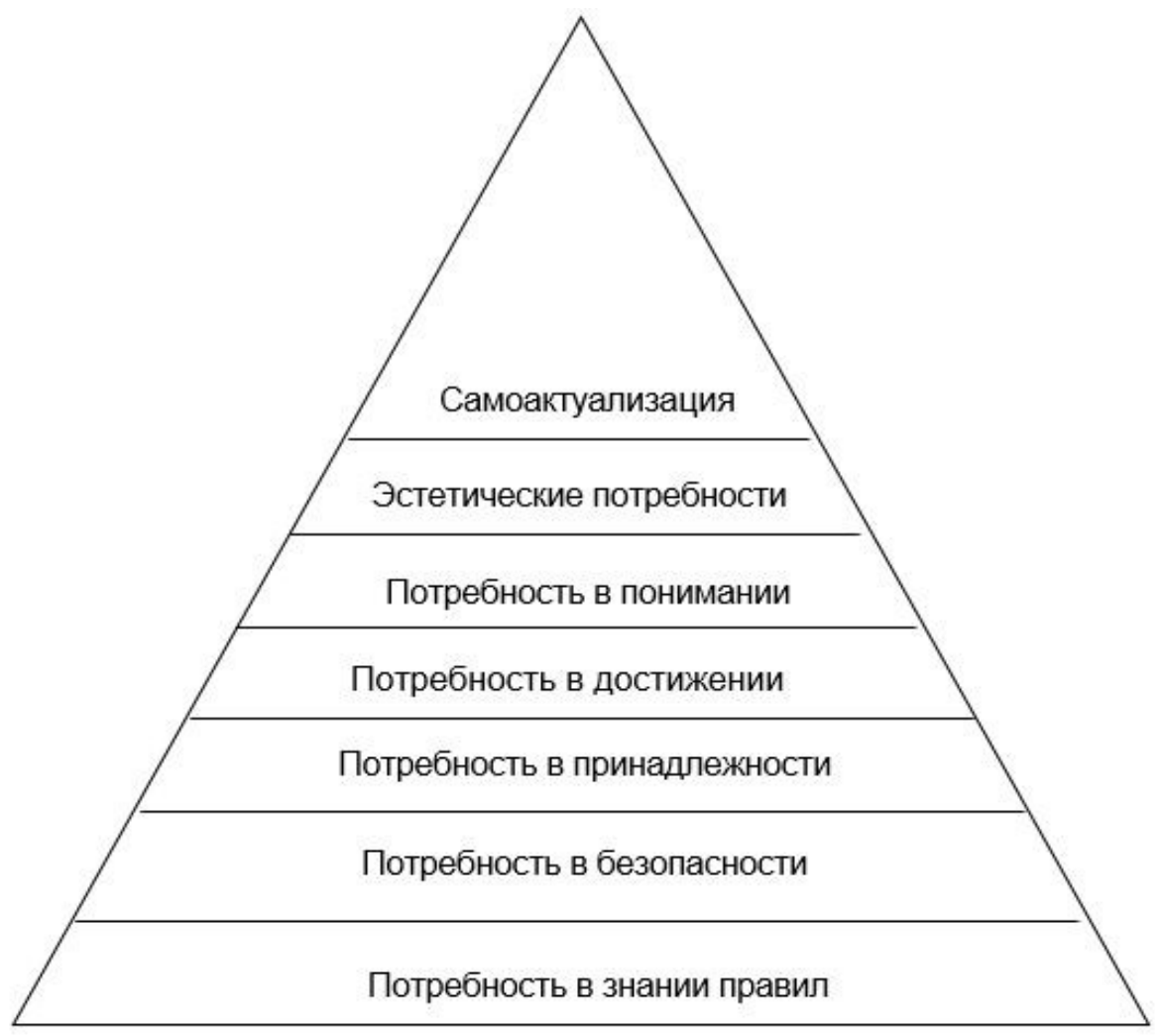

Рисунок 3 - Пирамидальная модель мотивации игроков по Сиангу

На нижнем уровне игроки ищут информацию, чтобы понять основные правила игры. Как только эта потребность будет удовлетворена, участники приобретают новую: в безопасности информации для сохранения шансов на победу.

Третий уровень соответствует потребности принадлежности к какой-либо группе игроков, в которой участники должны чувствовать себя комфортно в процессе игры и работать над достижением игровых целей.

После осознания того, что победа возможна, возникает потребность в ее достижении. На следующем уровне игроки начинают ожидать большего успеха, для этого они должны узнать еще больше об игре, например, о применении различных стратегий, ведущих к победе.

Шестой уровень - это эстетическая потребность, которая отражает необходимость в хорошей графике, визуальных эффектах, соответствующей музыке, звуковых эфффектах и т.д. Если речь идет о настольных играх, то мотивационные потребности здесь выражаются в наличии красивых комбинаций карт, обилии игровых карточек, фришек и бейджей.

Наконец, по достижению высшей ступени пирамидальной модели игроки хотят иметь возможность делать все, что угодно, но в рамках правил игры.

Согласно теории достижения, поведение человека направлено на развитие или демонстрацию - для себя или других - именно высоких, актуальных и нужных способностей, а не тех, показ которых будет вести к неудачам [9, с. 53]. Это означает, что в ситуациях, где можно достичь высоких результатов деятельности, люди желают себе успеха в максимально возможной степени. Все это указывает на высокую способность и стремление любой ценой избежать неудачи, которая может быть свидетельством наличия низкоуровневых способностей.

По мнению Аткинсона, добиться успеха и избежать неудачи - это два разных мотива человека. Они влияют на уровень сложности деятельности, которую люди выбирают для реализации. Индивиды с высокой мотивацией к успеху предпочитают задачи межпредметной сложности. Однако если мотив избегания неудачи более выражен, то люди предпочитают выбирать для исполнения либо очень простые, чтобы обеспечить себе гарантию выполнения полученного задания; либо очень трудные задачи, чтобы в случае неблагоприятного развития событий сослаться именно на высокий уровень сложности и высокий риск невыполнения задания [9, с. 60].

Почти во всех мотивационных играх визуализируются системы достижений и индикаторы состояния обеспеченности игрока. Эти системы призваны стимулировать игру и контролировать процесс достижений игроков. 
Согласно исследованию Монтолы, системы достижений - это структуры вознаграждения, обеспечивающие дополнительные цели для игроков, поэтому они провоцируют некоторую дружественную конкуренцию среди участников, основанную на сравнении себя с другими [10, с. 95]. Эти необязательные цели могут быть легкими, трудными, удивительными, забавными и достигаться индивидами в одиночку или в группе.

Разные категории достижений ориентированы на приобретение игроками конкретной выгоды. Незначительные достижения мотивируют игроков к дальнейшему изучению игры, предоставляют возможность проявить себя в получении более серьезных, бонусных достижений в игре, что значительно продлевает игровое время и позволяет участникам погрузиться в игровую ситуацию, а достижение определенного уровня мастерства служит в игре символом общественного статуса.

Многие игры предполагают возможность корректировки уровня сложности задачи с целью определения вероятности успеха и неудачи в соответствии с навыком игрока. Исследования показывают, что предложение выполнить задачу умеренной степени сложности повышает уровень мастерства игрока. Действительно, в большинстве игр предлагаемые задачи позволяют участникам относительно быстро и заметно прогрессировать [11].

Теория достижения потребности является основой для рассмотрения далее теории целеполагания, которая утверждает, что достижение конкретных сложных целей ведет к достижению более высокого результата [12, с. 214]. Цели влияют на производительность труда, направляя внимание, собирая усилия, повышая настойчивость и веру игроков в собственную способность выполнить поставленную задачу.

Соотношение между целями и способностью их достичь согласуется с условиями для появления у участников ощущения вовлеченности в игру. Главным определяющим фактором этого становится нахождение баланса между навыками игрока и целью самой игры.

Далее перейдем к анализу теорий, основанных на социальных потребностях игроков.

Теория социального сравнения и теория личных инвестиций рассматривают социальную сторону игр [13, с. 235]. Социальное сравнение состоит в том, что люди стремятся оценить свои убеждения, установки и способности в ряду подобных у окружающих. Теория личных инвестиций предполагает, что уровень, до которого человек будет вкладывать личные ресурсы, время и усилия в игровую ситуацию, зависит от личных стимулов, убеждений относительно себя и осознанных альтернатив.

Игры позволяют получить обратную связь в контексте сравнения себя с другими участниками. Игроки зарабатывают очки и упорядочиваются по рангу на основе общего их накопленного количества. Рейтинг может определяться количеством очков, набранных по отношению к самому статусному игроку, или в результате сравнения с другими игроками, находящимися в одинаковом тактическом положении по отношению к процессу игры. Сравнение игроков по количественным показателям провоцирует конкуренцию, которую можно представить как вызов к достижению новых целей и выполнению усложненных задач.

Процесс игры контролируется постоянным получением участниками оценок за игровые действия. Эти оценки включают в себя представление о том, как текущая позиция игрока соотносится с позициями других и какая тенденция ожидается в дальнейшем для развития конкуренции.

Таким образом, оценки обуславливаются результатами различных социальных сравнений, связанных с текущей игровой ситуацией. В соответствии с полученными оценками и социальными критериями самооценка каждого игрока будет подвергаться изменениям.

Теория личных инвестиций (PIT) интегрирует социальные влияния с исследованием мотивации достижения. Она состоит в том, что система ценностей, которую человек создает в форме убеждений, представлений, чувств, целей и задач, мотивирует его поведение. Эти когнитивные элементы являются ключом к пониманию и прогнозированию инвестиционного поведения индивида (участие, трата времени и усилий) [14, с. 113].

Концептуальная структура включает три основных сегмента: значение, предшествующие значения и личное инвестиционное поведение.

Теория определяет три основных компонента значения как наиболее важные для определения личных инвестиций в конкретных ситуациях: личные стимулы, чувство собственного достоинства и воспринимаемые варианты. Личные стимулы могут быть внутренними или внешними. К числу таких мотиваторов относятся стимулы выполнения заданий, отражающие повышение квалификации и мастерства; стимулы эгоизма, свидетельствующие о желании работать лучше по сравнению с другими; социальные стимулы, демонстрирующие солидарность с другими людьми, а также внешние вознаграждения в виде денежной компенсации или социального признания и одобрения со стороны наиболее авторитетных лиц. 
Игры используют все эти стимулы в качестве мотивационных крючков, которые поддерживают интерес и помогают обеспечить вовлеченность сотрудников в игровой процесс, а также вызвать желание повторно участвовать в игре. Как правило, стимулы зависят от некоторого аспекта достижения результата. Они могут быть выражены в виде очков, значков и репутации игрока. Большое разнообразие механизмов обратной связи помогает контролировать игровые решения участников и их поведение в соответствии с личной точкой зрения и поведением других игроков.

И, наконец, в правой части мотивационной модели внешняя сторона мотивации создается посредством внешних факторов, поощрений или стимулов. В этой связи необходимо рассмотреть две основных теории: теорию ожидаемой полезности и теорию подкрепления Скиннера.

Теория ожидаемой полезности связана с силой мотивации человека, его желанием стремиться к определенной цели, с возможностями ее достижения и с побудительной ценностью этой конкретной цели.

Предполагается, что ожидания и ценности индивида влияют на выбор достижений, настойчивость, прилагаемые усилия и производительность [15, с. 45]. Кроме того, нельзя не принимать в расчет специфические убеждения игроков относительно собственных способностей, субъективно воспринимаемого уровня сложности заданий и трудности достижения цели, предыдущий опыт и различные проявления социализации.

Рациональный игрок при выборе решения для выполнения задачи пытается максимизировать некоторую величину (благо); кажется естественным в качестве такой величины использовать математическое ожидание блага, появляющегося в результате избранного решения. Однако опыт показывает, что в реальной жизни многие игроки выбирают решение с меньшим математическим ожиданием, но и с меньшим риском.

Позволяя игрокам контролировать определенные аспекты своего игрового поведения, мы получаем возможность инвестировать личностные достижения в игровую среду и создавать идентификацию субъектов с каким-то аспектом внутри нее. Это приводит к тому, что игра может быть адаптирована к ситуации внутри организации, соотноситься с ее целями и стимулировать игроков развивать в себе те качества и тот потенциал, который в дальнейшем может быть использован и вне игровой среды для повышения эффективности профессионального самовыражения.

Макнамара предлагает два типа такого контроля [15, с. 49]. Первый - это предоставление определенной свободы контроля над различными аспектами окружающей игровой механики. Например, изменение цветовых схем, фона; выбор игровой ффишки или фригуры; определение выполняемой на данном этапе игры задачи. Второй тип - это постановка личных целей или подцелей. Например, можно определить для себя цель, состоящую в получении определенного количества баллов или в занятии верхней строчки в рейтинге. Макнамара также утверждает, что использование системы очков повышает мотивацию, обеспечивая четкую связь между усилиями в игре, эфффективностью и результатами [15, с. 52].

Теория подкрепления Скиннера, которую мы изложим далее, объясняет мотивацию к выполнению действий, которые приводят к внешним вознаграждениям. Скиннер утверждает, что поведение является продуктом внешнего подкрепления и различается в зависимости от способа его получения [16, с. 85].

Подкрепление в понимании Скиннера представляет собой результаты, которые усиливают вероятность ответа. С точки зрения ученого, непрерывное подкрепление обеспечивает желаемое поведение быстрее, чем частичное. В случае его устранения, наблюдается быстрая потеря достигнутого поведенческого результата. Частичное подкрепление, осуществляемое эпизодично, приводит к развитию большей устойчивости к вымиранию, чем непрерывное.

Завершая обзор существующих подходов и теорий, связанных с мотивацией участия в системах геймификации, стоит обратить внимание на следующее.

Глубокие теоретические познания - необходимое условие для грамотной работы любого менеджера по управлению персоналом и руководителя организации. Однако освоение профессии на основе анализа литературы, лекций, семинаров не дает практического опыта. Его приходится нарабатывать в процессе реальной трудовой деятельности методом проб и ошибок. Геймификация позволяет грамотно мотивировать персонал не только на достижение целей организации и повышение производительности труда (в том числе на рост прибыли, сохранение числа клиентов и привлечение новых), но и на сплочение коллектива, помогает определить социальную роль каждого работника и воспитывает чувство сопричастности к выполняемым задачам.

Руководитель имеет возможность приобрести уникальный опыт построения собственных систем мотивации, основанных на разработках геймификационных механик, дающих результат именно в конкретной организации и ее системе управления персоналом. В итоге он получает заинтересованного, лояльного специалиста, готового к полноценной работе. 


\section{Ссылки:}

1. Gamification: Using Game Design Elements in Non-Gaming Contexts / S. Deterding [et al.] // Proceedings of the 2011 Annual Conference Extended Abstracts on Human Factors in Computing Systems, CHI EA '11. N. Y., 2011. P. 2425-2428. https://doi.org/10.1145/1979742.1979575.

2. Muntean C.I. Raising engagement in e-learning through gamification // Proceedings 6th International Conference on Virtual Learning ICVL. Cluj-Napoca, 2011. P. 323-329.

3. Wu M. The gamification backlash + two long term business strategies [Электронный pecypc]. URL: http://lithosphere.lithium.com/t5/science-of-social-blog/The-Gamification- Backlash-Two-Long-Term-Business-Strategies/ba-p/30891 (дата обращения: 25.01.2014).

4. Bielik P. Integration and adaptation of motivational factors into software systems // Personalized Web-Science, Technologies and Engineering: 11th Spring PeWe Workshop Modra-Piesok, Proceedings. Bratislava, 2012. P. 31-32.

5. Maslow A.H. A theory of human motivation // Psychological Review. 1943. Vol. 50 (4). P. 370-396. https://doi.org/10.1037/h0054346.

6. Muntean C. I. Raising engagement in e-learning through gamification // Proceedings 6 th International Conference on Virtual Learning ICVL. Cluj-Napoca, 2011. P. 323-329.

7. Васильева Ж.Б. Мотивация участия в приложениях социальных сетей. Перспективы. М., 2012. С. 177-201.

8. Siang A.C., Rao R.K. Theories of learning: A computer game perspective // Proceedings of the IEEE Fifth International Symposium on Multimedia Software Engineering (ISMSE'03). Taichung, 2013. P. 239-245. https://doi.org/10.1109/mmse.2003.1254447.

9. Atkinson J.W., Litwin G.H. Achievement motive and test anxiety conceived as motive to approach success and motive to avoid failure // The Journal of Abnormal and Social Psychology. 1960. Vol. 60 (1). P. 52-63. https://doi.org/10.1037/h0041119.

10. Applying game achievement systems to enhance user experience in a photo sharing service / M. Montola [et al.] // Proceedings of the 13th International MindTrek Conference: Everyday Life in the Ubiquitous Era). Tampere, 2016. P. 94-97. https://doi.org/10.1145/1621841.1621859.

11. Hou J. Uses and gratifications of social games: Blending social networking and game play [Электронный ресурc] URL: http://firstmonday.org/article/ (дата обращения: 25.01.2014). https://doi.org/10.5210/fm.v16i7.3517.

12. Using social psychology to motivate contributions to online communities / K. Ling [et al.] // Proceedings of the 2004 ACM conference on Computer supported cooperative work. 2005. P. 212-221. https://doi.org/10.1145/1031607.1031642.

13. Wood J.V. Theory and research concerning social comparisons of personal attributes // Psychological Bulletin. 1989. Vol. 106 (2). P. 231-248. https://doi.org/10.1037/0033-2909.106.2.231.

14. Eccles J.S., Wigfield A. Motivational beliefs, values and goals // Annual Review of Psychology. 2012. Vol. 53 (1). P. $109-132$. https://doi.org/10.1146/annurev.psych.53.100901.135153.

15. McNamara D.S., Jackson G.T., Graesser A. Intelligent tutoring and games // Gaming for classroom-based learning: Digital role playing as a motivator of study Hershey, 2010. P. 44-65. https://doi.org/10.4018/978-1-61520-713-8.ch003.

16. Psychology: From inquiry to understanding / S.O. Lillienfeld [et al.]. Boston, 2019. P. 83-87.

Редактор: Ситникова Ольга Валериевна Переводчик: Кочетова Дарья Андреевна 